\title{
Comparative efficacy of lemborexant and other insomnia treatments: a network meta-analysis
}

Heather McElroy, MMedStat; Beth O'Leary, MPH, MSc; Michael Adena, PhD; Renee Campbell, MD, MBA, MPH; Amir Abbas Tahami Monfared, MD, PhD; and Genevieve Meier, PhD

\section{What is already known about this subject}

- Insomnia is common and associated with impaired daytime functioning and reduced health-related quality of life.

- Many current insomnia treatments such as benzodiazepines and benzodiazepine receptor agonists (Z-drugs) are associated with adverse consequences such as next-day impairment, confusion, and falls, especially in older people.

\section{What this study adds}

- This study places the efficacy and safety of lemborexant, a recently approved treatment for insomnia, in the context of other commonly used insomnia treatments.

- This study adds to previously published reviews in insomnia by undertaking a comparative network meta-analysis comparing lemborexant with multiple other insomnia treatments.

\section{Author affiliations}

Heather McElroy, MMedStat; Beth O'Leary, $\mathrm{MPH}, \mathrm{MSc}$; and Michael Adena, PhD, Datalytics, Kingston ACT, Australia. Renee Campbell, MD, MBA, MPH; Amir Abbas Tahami Monfared, MD, PhD; and Genevieve Meier, PhD, Eisai Inc., Woodcliff Lake, NJ.

\section{AUTHOR CORRESPONDENCE:}

Heather McElroy, +61 26113 1506;

heather@datalytics.com.a

J Manag Care Spec Pharm. 2021;27(9):1296-308

Copyright $\odot 2021$, Academy of Managed Care Pharmacy. All rights reserved.

subjective measures of WASO, TST, and SOL at 4 weeks (the differences were not statisti cally significant). No statistically significant interactions between treatment effect and older subpopulations were found, indicating that the treatment effect was similar in older and adult populations. The safety profile of lemborexant was broadly similar to the other treatments for SAEs and withdrawals due to $A E s$. A limitation is the age of some of the included studies (3 were published in 1990 or earlier). A further limitation is the lack of stratification of recommended doses. If the doses used in the study publications do not reflect doses used in clinical practice, this could potentially bias the results.

CONCLUSIONS: Lemborexant was ranked highest of the treatments studied on 3 out of the 4 objectively measured insomnia efficacy outcomes, with a safety profile broadly similar to other insomnia treatments. 
Insomnia is a common disorder involving sleep disturbances such as difficulty in falling asleep, difficulty maintaining sleep, or premature wakening, or a combination. Estimated prevalence in the United States varies from $3.9 \%$ to $27.3 \%$ and increases with age, reaching $30 \%$ to $48 \%$ in older adults. ${ }^{1-3}$

Insomnia may result in increased fatigue, reduced alertness, and impairment of daytime functioning. Lack of sleep is associated with increased risk of motor vehicle accidents and workplace accidents. ${ }^{4,5}$ Patients with insomnia reported high rates of car, workplace, and home accidents. ${ }^{6}$ In older people, sleep disturbance is associated with an increased risk of falls. ${ }^{7}$ Insomnia was associated with reduced quality of life in the United States, France, and Japan. ${ }^{8}$

Guidelines recommend cognitive behavioral therapy as first-line treatment for chronic insomnia..$^{9}$ If therapy is unavailable or ineffective, recommended pharmacological treatment include benzodiazepines (BZDs), benzodiazepine receptor agonists (also called Z-drugs [zolpidem, eszopiclone, zaleplon, zopiclone]), and some antidepressants, although some of these treatments are not indicated to treat insomnia. ${ }^{9}$ The first dual orexin receptor agonist, suvorexant, reported superior efficacy compared with placebo for subjective and objective sleep latency and sleep maintenance in two 3-month randomized controlled trials (RCT) and was approved in the United States in 2014..$^{10,11}$

Current treatments for insomnia may have unwanted effects including daytime sedation, rebound insomnia, confusion, and next-day impairment of complex tasks like driving. ${ }^{3}$ BZDs have potential for dependence and abuse. ${ }^{12}$ In older adults, BZDs increase the risks of memory impairment, falls, fractures, and avoidable hospital admissions, and Z-drugs can cause confusion and increase the risk of falls and fractures. ${ }^{12}$ It is suggested that BZDs and Z-drugs be avoided in older adults. ${ }^{3,12}$ Trazodone side effects include dizziness and orthostatic hypotension, which heighten the risk of falls and injuries. ${ }^{13}$ There is a need for long-term effective insomnia treatments with a low risk of adverse consequences and no dependency issues, especially in older populations.

Lemborexant is a new dual orexin receptor agonist recently approved in the United States, Japan, and Canada for the treatment of adult patients with insomnia characterized by difficulties with sleep onset and/or maintenance. ${ }^{14} \mathrm{It}$ has been evaluated in phase 3 clinical trials in comparison with placebo and zolpidem extended-release (ER) ${ }^{15,16}$ and improved sleep maintenance significantly compared with zolpidem-ER over 1 month of treatment (wake after sleep onset [WASO] in the second half of night vs zolpidem-ER -6.7 minutes, $P=0.004$ for lemborexant $5 \mathrm{mg}$ and $-8.0 \mathrm{~min}-$ utes, $\mathrm{P}<0.001$ for lemborexant $10 \mathrm{mg})^{16}$
Network meta-analysis (NMA) expands the scope of a conventional pairwise meta-analysis by analyzing simultaneously both direct comparisons of interventions within RCTs and indirect comparisons across RCTs. The RCTs are connected via a common comparator (eg, placebo). An NMA can thus estimate "indirectly" differences in outcomes between treatments that have never been directly compared, head-to-head, in RCTs. Previous meta-analyses in insomnia have been conducted ${ }^{17-23}$ however, only 1 included lemborexant, ${ }^{23}$ and this compared lemborexant with only 2 other treatments, suvorexant and zolpidem-ER. The objective of this systematic literature review and NMA was to compare lemborexant with 15 specified other insomnia treatments (derived from consultation with physicians) using measures of sleep maintenance, sleep onset, sleep quality, and incidence of adverse events (AEs).

\section{Methods}

\section{SEARCH STRATEGY}

To identify RCTs evaluating lemborexant or the 15 other treatments for insomnia, searches were conducted in Medline and Embase in October 2017 and updated in February 2019. Clinical trial registries were searched in February 2019 and again in March 2021. A targeted search of PubMed for RCTs of lemborexant or suvorexant was conducted in March 2021. The search strategy for Embase is provided in Supplementary Table 1 (available in online article).

The search strategy for BZD publications was limited to citations postdating a systematic review published in 2007. ${ }^{17}$ Relevant citations from this review were included manually.

The inclusion eligibility criteria were based on PICOTS (population, intervention, comparator, outcome, time frame, and study design):

Population: Primary insomnia in adults.

Intervention: Lemborexant and 15 other drug treatments (zaleplon, zolpidem [immediate release, (IR) or ER], zopiclone, eszopiclone, ramelteon, trazodone, suvorexant, brotizolam, flunitrazepam, etizolam, triazolam, alprazolam, estazolam, temazepam, or lorazepam), at doses recommended by the US Food and Drug Administration; European Union 5(France, Germany, Italy, Spain, and United Kingdom); or Pharmaceutical and Medical Devices Agency in Japan. Studies were included if at least 1 group was randomized to at least 1 of these interventions. Combination treatment was excluded.

Comparator: Other pharmacological treatments or placebo that allowed the study to be joined into the network. Exclusion at full-text review occurred if the comparator 
could not be used as a common reference to an intervention of interest.

Outcome: Latency to persistent sleep (LPS, objective), sleep onset latency (SOL, subjective), sleep efficiency (SE, objective and subjective), WASO (objective and subjective), total sleep time (TST, objective and subjective), Insomnia Severity Index (ISI), subjective quality of sleep (sQUAL), serious AEs (SAEs), withdrawal due to AEs, somnolence, dizziness, falls, or accidental injury.

Time frame: Treatment duration of at least 1 week.

Study design: RCT, excluding crossover design.

Other criteria included publication in English with a sample size of at least 50 patients in total across relevant treatment groups, and at least 20 patients evaluated in each relevant treatment group. Post hoc analyses, duplicate studies, or those reporting no usable data were excluded.

\section{SCREENING AND DATA EXTRACTION}

Studies identified in the search were initially screened using title and abstract by 2 independent reviewers, with disagreements resolved by consensus or a third reviewer. Full text was obtained for all potentially relevant articles. Publications were included after full-text review if they met the eligibility criteria and reported results for at least 1 outcome and at least 2 comparators of interest.

Data on study characteristics, interventions, comparators, results for outcomes of interest (point estimate and measure of variability), and quality assessment information were extracted from included publications by 2 independent reviewers using a standardized Microsoft Excel template. Any differences were resolved by consensus or a third person.

\section{OUTCOME MEASURES}

Efficacy outcomes assessed in insomnia trials include measures of sleep maintenance (TST, WASO, and SE), sleep onset (LPS, subjective SOL [sSOL]), and sleep quality (sQUAL). Some can be measured objectively using polysomnography (PSG; LPS), subjectively using a daily sleep diary recorded by the patient (sQUAL, sSOL), or both (TST, WASO, SE). TST is defined as total time from onset of persistent sleep to wakefulness. WASO is defined as the number of minutes in the wake stage after the onset of persistent sleep. SE is defined as TST divided by total time in bed, which for PSG studies is typically set at 8 hours, and thus TST and SE are essentially different measures of the same thing, particularly in PSG studies. LPS and sSOL are defined as the length of time that it takes to transition from full wakefulness to sleep. Insomnia severity was measured using the patient-completed ISI questionnaire. ${ }^{24}$ Safety outcomes included SAEs, withdrawals due to AEs, somnolence, dizziness, and falls or accidental injury. The primary time point for the NMA efficacy outcomes was 4 weeks from treatment initiation. For studies that did not report outcomes at 4 weeks, the measurement closest to 4 weeks was used, provided that it was between 1 and 6 weeks. Secondary efficacy time points were 3 months and 6 months (which included measurements up to 8 months).

Sleep quality was measured on multiple scales, ranging from 4-point scales to visual analogue scales running from 0 to 100 . The scales can also run in opposite directions, with higher scores indicating better sleep quality on some scales and worse on others. To analyze these data, all scales were transformed to a common scale of 1 to 10 , with 10 indicating better sleep quality. First, the direction was corrected so that a higher score indicated better quality of sleep for all studies by subtracting the mean from the maximum and adding to the minimum. Second, all scales were transformed to run from 1 to 10 using published methods. ${ }^{25}$

We combined dose levels within each study when patients were randomized to more than 1 of the approved doses of an intervention.

When not directly reported, the mean and SD were estimated from the median and range or interquartile range taking account of the skewness of the data. ${ }^{26}$ The standard error was derived from the SD, CI, test statistic, or P value. When no measure of variability was reported, the SD for the study was based on the weighted average SD reported in the other studies. ${ }^{27}$

\section{QUALITY ASSESSMENT}

Quality of evidence was assessed using the National Institute for Health and Care Excellence (NICE) methodology checklist for RCTs. ${ }^{28}$

\section{NETWORK META-ANALYSIS}

Bayesian NMA were performed at 4 weeks, 3 months, and 6 months, with placebo as the reference treatment because it was the most frequent comparator across the trials. A random-effects linear regression shared parameter model was fitted for the 4-week efficacy outcomes, ${ }^{29}$ and fixed-effects models were fitted for the 3 - and 6-month outcomes due to the sparseness of the networks. Efficacy estimates are reported as mean difference. These models also incorporated multi-arm trials under the assumption of homogenous between trial variance. Safety outcomes were analyzed as binary variables (number and percentage experiencing $\geq 1$ event), without continuity correction and estimates reported as odds ratio (OR). Complementary log-log (cloglog) models using duration of treatment as an offset were also fitted as sensitivity analysis with estimates reported as hazard ratios. 
Trials with no events across all treatment groups of interest were excluded, as these do not provide evidence of the direction or magnitude of the treatment effect, and may dilute treatment estimates, which is inappropriate for detecting safety signals. Trials with no events in 1 group and events in another group were included where possible. All estimates were summarized using median with 95\% credible intervals (CrI). The probability each intervention was ranked first (best), second, third, and so on, was estimated from the model for each outcome and displayed in rankograms.

Model parameters were estimated using the Markov Chain Monte Carlo method in WinBUGS version 1.4.3 (The BUGS Project). ${ }^{30}$ Models were developed using NICE guidance. ${ }^{29}$ Three chains were run for 50,000 iterations and discarded as "burn-in," and then the model was run for a further 100,000 iterations for inference. Convergence was assessed by visual inspection of the history plot and Gelman-Rubin diagnostics. Noninformative priors were used. The residual deviance Dres was compared with the number of datapoints to assess model fit, and $\tau$ (tau), an estimate of the SD of underlying true effects across studies, is presented as a measure of heterogeneity for random effects models.

\section{ASSESSMENT OF CONSISTENCY}

For some comparisons, there was direct evidence (when there were RCTs that included both treatments) as well as indirect evidence (where there were RCTs that included 1 of the treatments and a common reference treatment, typically placebo). We used the network suite of commands in Stata (StataCorp) to examine the consistency of direct versus indirect evidence for networks with closed loops. ${ }^{31-33}$

\section{ASSESSMENT OF BIAS}

Reporting bias or small study bias ("publication bias") was evaluated by visually assessing comparison-adjusted funnel plots for endpoints reported by more than 10 studies. ${ }^{33,34}$

\section{SUBGROUP ANALYSIS}

Subgroup analysis was conducted for older adults. Subgroup analyses incorporated the proportion of the study population defined as older (ranging from 0\% for studies not containing older adults to $100 \%$ for studies only containing older adults). Older adults were generally defined as people aged at least 65 years, although definitions varied by study. Subgroup analyses were conducted by fitting an interaction model under the assumption of the same interaction for all active treatments because neither unrelated nor related interaction models were feasible given the sparse networks. ${ }^{35}$
The interaction model fitted means that only 1 interaction term is estimated for active treatment versus placebo, and thus cancels out when comparing 2 active treatments. This may not be an appropriate assumption if, for example, some active treatments work better in the older subgroup and others in the adult subgroup. The plan was to fit individual models for the older network and the adult network separately. However, due to the sparseness of the networks, these estimates were not robust.

\section{Results}

\section{SEARCH RESULTS}

Figure 1 summarizes the results of the literature searches and the screening/selection process conducted in February 2019 to identify RCTs of the treatments of interest. No additional trials were identified in the targeted searches in March 2021. A total of 45 studies were included. 111,16,36-77

\section{STUDY CHARACTERISTICS}

The 45 studies covered lemborexant and 15 other insomnia treatments, of which the most commonly studied were zolpidem-IR or -ER. No studies of etizolam, alprazolam, or lorazepam met our inclusion criteria. The studies covered a long period (1984 to 2020). Most were placebo controlled and all were double blind (Supplementary Table 2, available in online article).

\section{QUALITY ASSESSMENT}

The quality assessment results are summarized in Supplementary Table 3 (available in online article). Generally, the older studies tended to report less detail.

\section{EFFICACY OUTCOMES}

Objective sleep outcomes measured by PSG were analyzed at 4 weeks and subjective outcomes at 4 weeks, 3 months, and 6 months. The number of studies and interventions varied by outcome, with subjective outcomes more frequently reported (Table 1). The network diagram for TST is shown in Figure 2. The other efficacy network diagrams are presented in Supplementary Figures 1-4 and results of individual studies in Supplementary Tables 4-13 (supplementary materials available in online article).

Of the objective efficacy outcomes, lemborexant was the only treatment statistically significantly superior to placebo on TST and was also statistically significantly superior to ramelteon, prolonging sleep by an average of 32 minutes (Table 1). Lemborexant was statistically significantly superior to placebo, zolpidem-IR, and ramelteon for WASO (average reduction of 20-25 minutes), and to placebo, zolpidem-ER, zaleplon, and triazolam for LPS (13-23 minutes). All 


\section{FIGURE 1 PRISMA Flow Diagram}

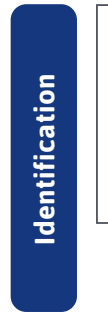

Additional records identified through other sources

$$
(n=6)^{a}
$$

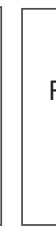

Records identified from Buscemi et al, 2007 $(n=14)$

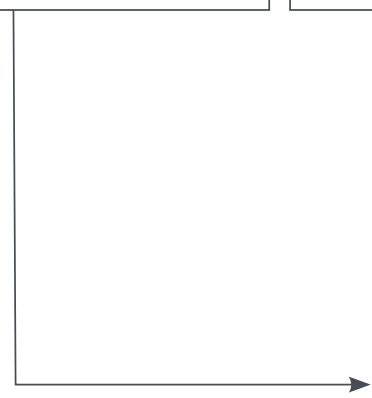

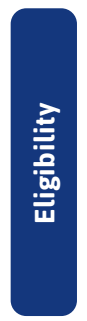

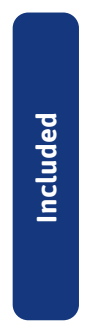

Records identified through database searching, 2017 $(\mathrm{n}=1,374)$

Records after duplicates removed $(n=1,152)$
Records identified through database searching, 2019 $(n=578)$

Records after duplicates removed $(n=543)^{b}$
Records excluded by title/abstract

$$
(n=1,642)
$$

Reasons for exclusion:

Records screened by title/abstract $(n=1,715)$

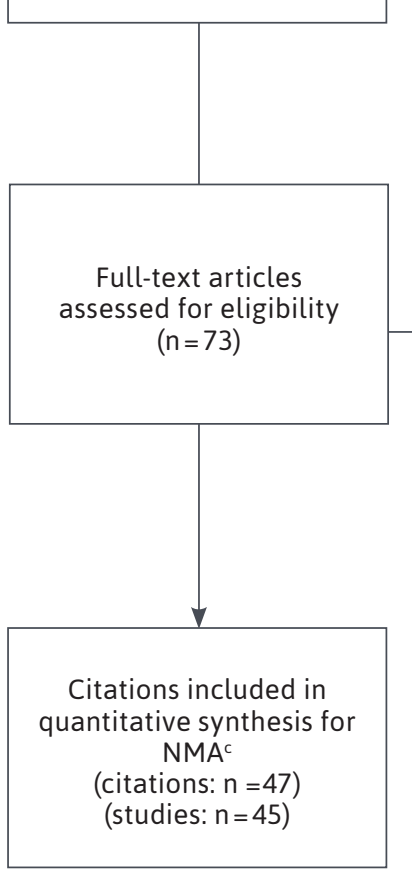

(A) Not an RCT $(\mathrm{n}=917)$

(B) Not primary insomnia $(\mathrm{n}=259)$

(C) Not comparators of interest $(n=124)$

(D) Small sample size $(\mathrm{n}=85)$

(E) Pediatric patients $(\mathrm{n}=2)$

(F) Post hoc analysis or duplicate $(n=159)$

(G) Crossover studies $(\mathrm{n}=30)$

(H) Not in English $(n=30)$

(I) Intermittent or not approved dose $(n=24)$

(J) Did not include outcome of interest $(n=11)$

(K) Treatment length $<1$ week $(n=1)$

Records excluded by full text

$$
(n=26)
$$

Reasons for exclusion:

(A) Small sample size $(n=4)$

(B) Did not include outcome of interest $(n=12)$

(C) Not primary insomnia $(\mathrm{n}=3)$

(D) Treatment length $<1$ week $(n=1)$

(E) Not a comparator of interest $(n=5)^{d}$

(F) Intermittent or not approved dose $(n=1)$

Note: Searches of databases from inception through February 2019 are shown. Targeted searches conducted in March 2021 did not identify any new RCTs. aSix records were identified by manual searching: 1 article (Ancoli-Israel) was identified from review of the reference list of Ancoli-Israel 36,$37 ; 1$ unpublished study (TL020) was identified from a ramelteon review (Kuriyama A, Honda M, Hayashino Y. Ramelteon for the treatment of insomnia in adults: a systematic review and meta-analysis. Sleep Med. 2014;15[4]:385-92); 3 CSRs for lemborexant (Studies 201, 303, and 304); and 1 poster of Study 303.61

${ }^{6}$ There were 107 records from the updated search of Embase.com (including Embase and Medline) and 436 from clinical trial registries.

'A total of 47 articles/reports on 45 distinct studies were identified for inclusion. Note that 1 article, Herring, ${ }^{11}$ reported 2 trials; Study 201 is represented by 2 citations (the CSR and the publication by Murphy ${ }^{60}$ ); Study 303 is represented by 2 citations (the CSR and the poster by Yardley ${ }^{61}$ ); and Study 304 is represented by 2 citations (the CSR and the abstract/poster by Rosenberg ${ }^{80}$ ). Studies 303 and 304 were subsequently published as full manuscripts after the date of the search, ${ }^{14,16}$ and the full manuscripts are cited in this review instead of the earlier posters.

${ }^{d}$ These studies were excluded at full-text review as not a comparator of interest if there was no opportunity to use that comparator as a common reference to a drug of interest. This applied to midazolam, nitrazepam, propiomazine, and pentobarbitone.

$C S R=$ clinical study report; NMA = network meta-analysis; $P R I S M A=$ preferred reporting items for systemic reviews and meta-analyses; $R C T=$ randomized controlled trial. 


\section{TABLE 1 Comparison of Lemborexant With Other Treatments: Efficacy Results at 4 Weeks}

\begin{tabular}{|c|c|c|c|c|c|c|c|c|c|}
\hline \multirow[b]{2}{*}{$\begin{array}{l}\text { Lemborexant } \\
\text { vs comparator }\end{array}$} & \multicolumn{4}{|c|}{ Objective (PSG) } & \multicolumn{5}{|c|}{ Subjective } \\
\hline & TST, min & WASO, $\min$ & LPS, min & SE, \% & sTST, $\min$ & sWASO, $\min$ & sSOL, $\min$ & $\begin{array}{c}\text { ISI } \\
\text { (scale } 0-28)\end{array}$ & $\begin{array}{c}\text { sQUAL } \\
\text { (scale 1-10) }\end{array}$ \\
\hline Placebo & $\begin{array}{c}38.1 \\
(16.3,60.7)\end{array}$ & $\begin{array}{c}-21.3 \\
(-29.6,-10.1)\end{array}$ & $\begin{array}{c}-18.6 \\
(-29.0,-10.9)\end{array}$ & $\begin{array}{c}7.2 \\
(3.4,10.8)\end{array}$ & $\begin{array}{c}22.5 \\
(12.6,32.7)\end{array}$ & $\begin{array}{c}-10.9 \\
(-19.0,-3.4)\end{array}$ & $\begin{array}{c}-13.2 \\
(-20.2,-6.0)\end{array}$ & $\begin{array}{c}-1.9 \\
(-3.3,-0.5)\end{array}$ & $\begin{array}{c}0.3 \\
(0.1,0.6)\end{array}$ \\
\hline Suvorexant & NA & $\begin{array}{c}4.1 \\
(-8.7,19.4)\end{array}$ & $\begin{array}{c}-9.4 \\
(-23.4,1.8)\end{array}$ & NA & $\begin{array}{c}4.0 \\
(-9.8,18.1)\end{array}$ & $\begin{array}{c}-4.0(-15.7 \\
6.9)\end{array}$ & $\begin{array}{c}-7.1 \\
(-18.5,4.4)\end{array}$ & $\begin{array}{c}-0.6 \\
(-2.7,1.6)\end{array}$ & $\begin{array}{c}-0.1 \\
(-0.5,0.3)\end{array}$ \\
\hline Zolpidem-IR & $\begin{array}{c}21.1 \\
(-13.0,50.2)\end{array}$ & $\begin{array}{c}-19.6 \\
(-31.9,-0.3)\end{array}$ & $\begin{array}{c}-6.3 \\
(-19.7,3.8)\end{array}$ & $\begin{array}{c}3.3 \\
(-1.8,9.0)\end{array}$ & $\begin{array}{c}-5.3 \\
(-17.2,6.3)\end{array}$ & $\begin{array}{c}3.1 \\
(-7.4,13.3)\end{array}$ & $\begin{array}{c}0.2 \\
(-8.0,8.9)\end{array}$ & NA & $\begin{array}{c}-0.3 \\
(-0.6,0.0)\end{array}$ \\
\hline Zolpidem-ER & $\begin{array}{c}22.6 \\
(-4.6,51.0)\end{array}$ & $\begin{array}{c}-7.2 \\
(-16.8,4.6)\end{array}$ & $\begin{array}{c}-13.4 \\
(-24.4,-4.9)\end{array}$ & $\begin{array}{c}4.6 \\
(-0.9,9.8)\end{array}$ & $\begin{array}{c}-2.1 \\
(-14.6,10.3)\end{array}$ & $\begin{array}{c}8.1 \\
(-2.6,18.1)\end{array}$ & $\begin{array}{c}-8.0 \\
(-17.2,1.0)\end{array}$ & $\begin{array}{c}0.3 \\
(-1.8,2.5)\end{array}$ & $\begin{array}{c}-0.2 \\
(-0.5,0.2)\end{array}$ \\
\hline Zopiclone & $\begin{array}{c}24.2 \\
(-21.7,73.1)\end{array}$ & NA & $\begin{array}{c}-11.1 \\
(-29.6,4.8)\end{array}$ & $\begin{array}{c}4.4 \\
(-0.1,8.8)\end{array}$ & NA & NA & NA & $\begin{array}{c}0.9 \\
(-2.4,4.0)\end{array}$ & NA \\
\hline Eszopiclone & $\begin{array}{c}10.0 \\
(-25.6,48.2)\end{array}$ & $\begin{array}{c}-8.7 \\
(-21.8,7.1)\end{array}$ & $\begin{array}{c}-6.0 \\
(-20.1,5.6)\end{array}$ & $\begin{array}{c}1.8 \\
(-2.9,6.5)\end{array}$ & $\begin{array}{c}-12.3 \\
(-24.3,0.2)\end{array}$ & $\begin{array}{c}3.6 \\
(-6.0,12.7)\end{array}$ & $\begin{array}{c}4.4 \\
(-5.1,13.6)\end{array}$ & $\begin{array}{c}1.3 \\
(-0.6,3.1)\end{array}$ & $\begin{array}{c}-0.6 \\
(-0.9,-0.2)\end{array}$ \\
\hline Zaleplon & $\begin{array}{c}31.1 \\
(-0.5,63.9)\end{array}$ & NA & $\begin{array}{c}-14.5 \\
(-29.7,-1.6)\end{array}$ & NA & $\begin{array}{c}13.0 \\
(0.0,25.6)\end{array}$ & NA & $\begin{array}{c}0.5 \\
(-8.7,9.9)\end{array}$ & NA & $\begin{array}{c}0.1 \\
(-0.2,0.5)\end{array}$ \\
\hline Trazodone & NA & NA & NA & NA & $\begin{array}{c}2.9 \\
(-17.4,23.0)\end{array}$ & $\begin{array}{c}-1.2 \\
(-17.1,14.0)\end{array}$ & $\begin{array}{c}-5.0 \\
(-20.3,10.6)\end{array}$ & NA & $\begin{array}{c}-0.2 \\
(-0.8,0.4)\end{array}$ \\
\hline Flunitrazepam & NA & NA & NA & NA & NA & NA & NA & NA & NA \\
\hline Estazolam & NA & NA & NA & NA & NA & NA & NA & NA & $\begin{array}{c}-0.5 \\
(-1.0,0.0)\end{array}$ \\
\hline Triazolam & $\begin{array}{c}22.1 \\
(-16.4,62.1)\end{array}$ & NA & $\begin{array}{c}-23.2 \\
(-38.8,-9.6)\end{array}$ & $\begin{array}{c}5.3 \\
(-1.4,12.1)\end{array}$ & $\begin{array}{c}-3.7 \\
(-21.2,13.6)\end{array}$ & NA & $\begin{array}{c}2.0 \\
(-14.4,18.5)\end{array}$ & NA & $\begin{array}{c}-0.4 \\
(-1.1,0.3)\end{array}$ \\
\hline Temazepam & NA & NA & NA & NA & $\begin{array}{c}10.5 \\
(-7.9,29.2)\end{array}$ & $\begin{array}{c}3.8 \\
(-14.5,21.9)\end{array}$ & 1.2 & NA & NA \\
\hline Brotizolam & NA & NA & NA & NA & $\begin{array}{c}-35.7 \\
(-76.4,5.7) \\
\end{array}$ & $\begin{array}{c}6.8 \\
(-14.6,28.2)\end{array}$ & NA & NA & NA \\
\hline Ramelteon & $\begin{array}{c}31.7 \\
(1.3,62.8)\end{array}$ & $\begin{array}{c}-24.8 \\
(-40.8,-6.0)\end{array}$ & $\begin{array}{c}-8.0 \\
(-20.7,3.0)\end{array}$ & $\begin{array}{c}5.8 \\
(-0.9,12.3)\end{array}$ & $\begin{array}{c}15.4 \\
(3.6,27.3)\end{array}$ & $\begin{array}{c}-16.9 \\
(-33.3,-1.1)\end{array}$ & $\begin{array}{c}-6.9 \\
(-15.5,2.0)\end{array}$ & NA & $\begin{array}{c}0.3 \\
(-0.1,0.6)\end{array}$ \\
\hline$\tau(\operatorname{tau})$ & $\begin{array}{c}11.0 \\
(1.1,26.9)\end{array}$ & $\begin{array}{c}3.7 \\
(0.2,14.1)\end{array}$ & $\begin{array}{c}4.2 \\
(0.4,10.2)\end{array}$ & $\begin{array}{c}1.3 \\
(0.1,8.0)\end{array}$ & $\begin{array}{c}4.7 \\
(0.6,9.0)\end{array}$ & $\begin{array}{c}3.9 \\
(0.3,9.8)\end{array}$ & $\begin{array}{c}5.3 \\
(3.2,8.1)\end{array}$ & $\begin{array}{c}0.8 \\
(0.2,2.2)\end{array}$ & $\begin{array}{c}0.1 \\
(0.0,0.3)\end{array}$ \\
\hline $\begin{array}{l}\text { Dres vs number of } \\
\text { datapoints }\end{array}$ & 21.6 vs 21 & 21.4 vs 20 & 39.4 vs 36 & 25.5 vs 22 & 72.0 vs 73 & 49.9 vs 45 & 73.6 vs 74 & 21.0 vs 21 & 56.7 vs 57 \\
\hline Number of studies & 10 & 11 & 18 & 11 & 34 & 22 & 34 & 10 & 27 \\
\hline Number of treatments & 9 & 7 & 10 & 8 & 12 & 10 & 11 & 6 & 11 \\
\hline Number of participants & 2,974 & 3,663 & 4,822 & 2,800 & 13,370 & 7,989 & 13,923 & 5,257 & 10,603 \\
\hline \multicolumn{10}{|c|}{$\begin{array}{l}\text { Note: Data are the mean difference between lemborexant and each comparator reported as median ( } 95 \% \text { CrI) from random effects NMA. sQUAL is reported on a } \\
\text { scale from } 1 \text { to } 10 \text {, where higher score indicates better quality of sleep. ISI is measured on a scale from } 0 \text { to } 28 \text {, where lower scores indicate lower severity. Thus for } \\
\text { TST, SE, and sQUAL, positive differences indicate lemborexant performs better; for WASO, LPS/sSOL, and ISI, negative difference indicates lemborexant performs } \\
\text { better. Statistically significant differences ( } 95 \% \text { Crl excluding 0) are highlighted in bold. }\end{array}$} \\
\hline \multicolumn{10}{|c|}{ 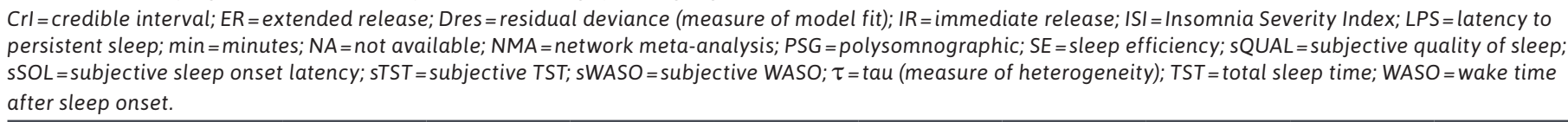 } \\
\hline
\end{tabular}

other comparisons showed no statistically significant differences versus lemborexant. Pairwise comparisons for other comparators and placebo are presented in Supplementary Tables 14-17 (available in online article).
Lemborexant had the highest probability of being the best treatment for 3 of the 4 objective efficacy outcomes (TST, LPS, and SE) and was ranked second to suvorexant on WASO at 4 weeks (Figure 3). Rankograms for other 


\section{FIGURE 2 Network Diagram for Included Studies Reporting TST (PSG) at 4 Weeks}

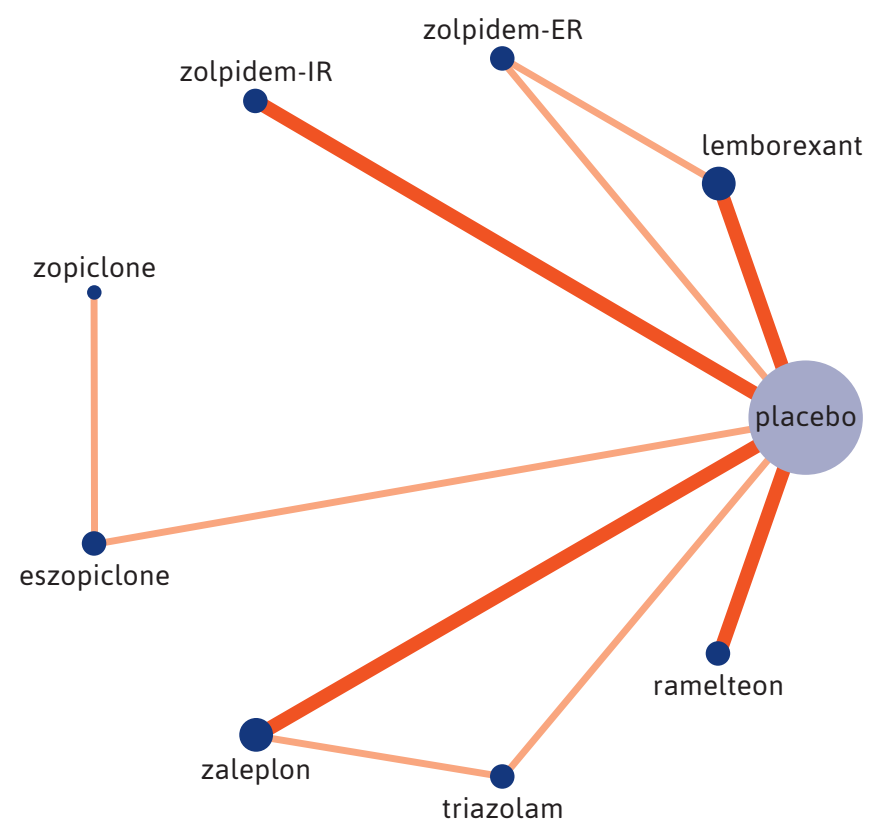

Note: Light orange lines join treatment pairs that occur in 1 study, and dark orange lines join treatment pairs that occur in 2 studies. The size of each circle is proportional to the number of treatment comparisons that involve that treatment.

$E R=$ extended release; $I R=$ immediate release; $P S G=$ polysomnographic; TST = total sleep time.

treatments are in Supplementary Figures 5 and 6 (available in online article).

Rankograms for the subjective efficacy outcomes at 4 weeks are shown in Supplementary Figures 7-9 (available in online article). Eszopiclone was highly ranked for sSOL and ISI at 4 weeks (Supplementary Figure 7), although it was not statistically significantly different from lemborexant on either outcome (Table 1). Lemborexant was rated more highly than suvorexant for subjective WASO (sWASO), subjective TST (STST), or sSOL at 4 weeks, although the differences were not statistically significant (Table 1).

Eszopiclone was highly ranked for sSOL and ISI at 3 months and 6 months, although sSOL was not statistically significantly different from lemborexant at either time point (Supplementary Table 18). At 3 months, lemborexant was statistically significantly superior to suvorexant on sSOL and to ramelteon on sWASO (Supplementary Table 18). At 6 months, lemborexant was statistically significantly superior to ramelteon on sTST and sWASO (Supplementary Table 18). Pairwise comparisons for other comparators and placebo are presented in Supplementary Tables 19-33 (all available in online article).

\section{SAFETY OUTCOMES}

Network diagrams for the safety outcomes are shown in Supplementary Figure 10, and results of individual studies are shown in Supplementary Tables 34-38 (available in online article). The incidence of SAEs was not statistically significantly different between lemborexant and placebo, zolpidem-ER, eszopiclone, or ramelteon, and was statistically significantly smaller for suvorexant (OR 5.48, 95\% CrI=1.26-32.06; Table 2). Data were sparse for this endpoint (12 out of 45 studies). There were no statistically significant differences between lemborexant and other treatments for withdrawals due to AEs (Table 2). There were no statistically significant differences for falls/injuries, although data were sparse as 8 trials reported a fall/injury, and 3 trials reported no falls.

Lemborexant was associated with statistically significantly lower incidence of dizziness compared with zolpidem-IR, zolpidem-ER, and eszopiclone (Table 2). Lemborexant was associated with a statistically significantly higher rate of somnolence compared with placebo, suvorexant, zolpidem-IR, zolpidem-ER, eszopiclone, zaleplon, estazolam, and ramelteon (Table 2). Pairwise comparisons for other comparators and placebo are presented in Supplementary Tables 39-43 (available in online article).

Withdrawal due to AEs, the proportion experiencing at least $1 \mathrm{SAE}$, fall, or accidental injury increased with trial duration. Nonetheless, similar results were observed when duration was taken into account (Supplementary Table 44, available in online article). This is not unexpected, as the duration was assumed to be the same for each group within a trial and thus has little influence on the estimated ratios.

\section{OLDER SUBPOPULATION}

Ten studies were designed to assess insomnia treatment in older adults, and a further 25 studies were conducted in adult patients. The remaining 10 studies, while including older and adult patients, did not report results by subgroup.

Supplementary Table 45 (available in online article) presents the $\beta$ value for interaction for each outcome analyzed in the older subgroup. No statistically significant interactions between treatment effect and older subpopulation were found for any efficacy outcomes, indicating that the treatment effect was similar in older and adult populations.

There was some evidence of an interaction for withdrawals due to AEs (OR 0.59, 95\% CrI=0.35-1.01), SAEs (OR 0.52, 95\% CrI = 0.08-3.02), and falls / injury (OR 2.89, 95\% CrI = 0.6915.75), but these were not statistically significant. There was a statistically significant interaction between age and 


\section{FIGURE 3 Ranking of Lemborexant on Objective Efficacy Outcome Measures at 4 Weeks}

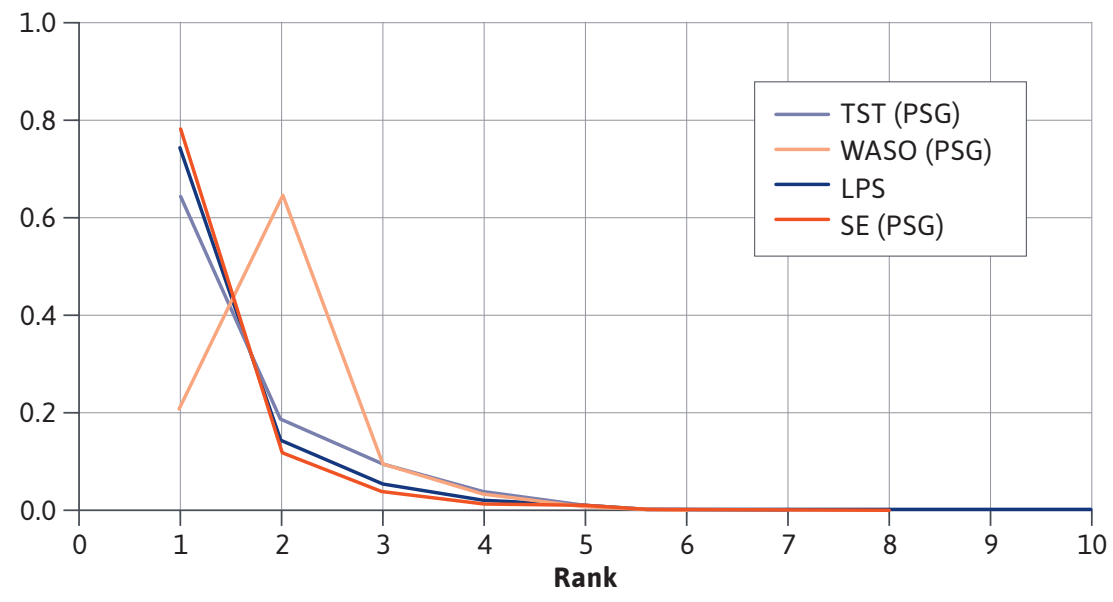

LPS = latency to persistent sleep; PSG = polysomnographic; $S E=$ sleep efficiency; TST = total sleep time; WASO = wake after sleep onset.

treatment effect for somnolence (OR $0.48,95 \% \mathrm{CrI}=0.26-0.87)$. However, separate analyses of studies in older adults (based on 17 of the 23 studies that reported somnolence; 7 older and 10 adult) indicated that the assumption of the same interaction for all active treatments may not be reasonable in this case. Older people appeared to have high odds of somnolence with zolpidem-IR and zaleplon, but lower odds with zolpidem-ER, eszopiclone, and ramelteon (all vs placebo, data not shown).

\section{EXPLORATION OF HETEROGENEITY, BIAS, AND CONSISTENCY}

Heterogeneity $(\tau)$ was small compared with the treatment effects versus placebo (Table 1), indicating that the estimates are robust. Residual deviance indicated a good model fit (Table 1).

Safety was not consistently reported across studies, with few studies available for analysis of SAEs (12 of 45) or falls/accidental injury (8 of 45). Small study bias was examined through comparison-adjusted funnel plots for 4-week efficacy and safety endpoints reported by more than 10 studies (Supplementary Figures 11-13, available in online article). There was no evidence of asymmetry (small study effects) for any efficacy endpoints, but a suggestion of some asymmetry for safety endpoints (withdrawals due to AEs, somnolence, and dizziness).

There was no evidence of inconsistency for TST, WASO, sTST, sWASO, sSOL, ISI, or sQUAL at 4 weeks (global test $\mathrm{P}>0.20$ for all). However, there was evidence of inconsistency for LPS $(P=0.002)$ and SE $(P=0.011)$, which is likely to be primarily due to the small Ware study ${ }^{74}$ which reported nonsignificant superiority of placebo over zolpidem-IR. There was no evidence of inconsistency based on the global test $(P>0.05)$ for the safety outcomes assessed, although there was some evidence of inconsistency in the placebo-zolpidem-IR-temazepam and placebo-zolpidem-IR-triazolam loops for withdrawals due to AEs.

\section{Discussion}

This NMA examined the efficacy and safety of lemborexant and 15 other insomnia treatments across 45 studies. Only 1 previous limited meta-analysis had included lemborexant, ${ }^{23}$ and only included 3 interventions (lemborexant, suvorexant, and zolpidem-ER). The NMA concluded that lemborexant $10 \mathrm{mg}$ had superior efficacy ${ }^{23}$ which is consistent with our more comprehensive finding that lemborexant ranked highest on 3 of the 4 objective measures (TST, LPS, and $\mathrm{SE})$. In our analysis, the highest-ranked intervention exceeded clinical significance thresholds for mean change relative to placebo proposed by the American Academy of Sleep Medicine for PSG-measured TST, LPS, SE, and WASO, ${ }^{21}$ indicating that the results should potentially translate into clinical benefit to patients.

The safety profile of lemborexant was broadly similar to other treatments with respect to SAEs and withdrawals due to AEs, and lemborexant was associated with statistically significantly less dizziness than zolpidem-IR, zolpidem-ER, and eszopiclone. There was a significant difference in SAEs for lemborexant versus suvorexant; however, this may be due to the high placebo rate for SAEs in suvorexant trial 1, where the SAE rate was $2.9 \%$ for placebo versus $0.4 \%$ for suvorexant, ${ }_{11}^{11}$ rather than a difference between lemborexant and suvorexant per se. Lemborexant was also associated with increased odds of somnolence. More somnolence was observed with higher lemborexant doses. ${ }^{14}$ However, there was no statistical difference in objective measures such as reaction time between lemborexant and placebo or other assessments of the potential for residual morning sleepiness, ${ }^{60,78}$ indicating that lemborexant was not associated with clinically meaningful residual morning sleepiness. 


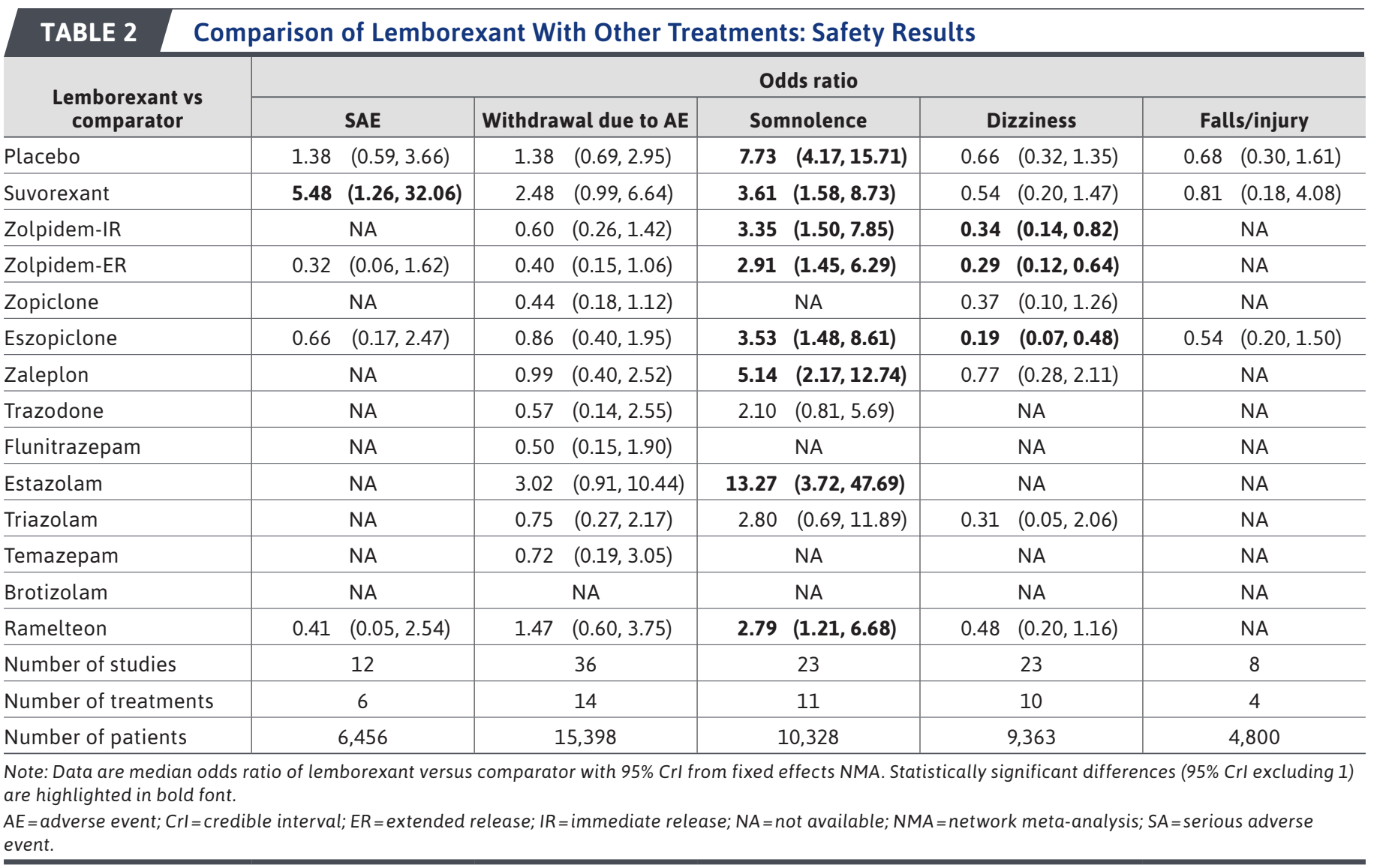

No statistically significant interactions were found between treatment effect and older subpopulation, indicating that the treatment effect was similar in older and adult populations. Older patients are an important subgroup, as insomnia is common in older people, and some current treatments have warnings and precautions related to their use in older populations (eg, eszopiclone).$^{79}$ However, the sparse networks limited the interaction models that could be fitted. As a result, although the subgroup analysis indicated that the results from the main NMA also apply to the older population, comparisons between different active treatments in older adults could not be made.

This NMA has a number of strengths. It included both objective endpoints measured by PSG (permitting consistent measurement) and subjective outcomes (reflecting the patient's perception of poor sleep in a real-life setting). Clinical guidelines include both subjective and objective measures as critical outcomes with defined clinical significance thresholds. ${ }^{21}$ The low heterogeneity and the consistency observed for most outcomes support confidence in the robustness of the NMA results. Similarly, the funnel plot analysis did not identify substantial publication bias.

\section{LIMITATIONS}

This analysis has a number of limitations. The included studies were published over 3 decades (1984-2020), and earlier studies were conducted when there were fewer options for treating insomnia and lower quality of trial reporting. Some studies failed to report numerical results when the results were not statistically significant, and therefore could not be included. This could potentially lead to publication bias as the nonstatistically significant differences might have diluted any treatment effect had it been possible to include them.

Not all trials included the same subjective and objective measurements, resulting in limitations in comparing across all drugs on each measurement.

The analysis used approved insomnia doses and averaged results across all doses, as is standard practice. 
However, if the doses used in the publications do not reflect those used in clinical practice, this could potentially bias the results. The starting dose of lemborexant is $5 \mathrm{mg},{ }^{15}$ and the lemborexant phase 3 studies in the NMA included equal numbers of patients receiving $5 \mathrm{mg}$ and $10 \mathrm{mg}$. In contrast, the starting dose for suvorexant is $10 \mathrm{mg},{ }^{10}$ but data were only available for suvorexant at a dose of $15 \mathrm{mg}$ (older adults) and $20 \mathrm{mg}$ (adults). Most patients in the long-term eszopiclone studies received $3 \mathrm{mg}$, whereas there is a warning of impaired daytime function for doses of $2 \mathrm{mg}$ and $3 \mathrm{mg}$, and a dose of greater than $2 \mathrm{mg}$ is contraindicated in older patients. ${ }^{79}$ Lower initial doses are recommended in women than in men for zolpidem-IR or -ER. If doses in some of the studies in the NMA did not reflect recommendations, this could potentially have biased the efficacy results.

The time point of the efficacy outcomes included in the 4-week analysis could vary from 1 to 6 weeks.

To perform the analysis, the different scales used to measure sleep quality were transformed to a common scale. This has some limitations, as the question about sleep quality may have differed, and the transformation does not guarantee comparability of the categories in the transformed scales (eg, data from a 4-point scale are less precise than from a scale with more categories). sQUAL is therefore less robust than the other efficacy outcomes, which have standardized definitions and measurement.

The level of evidence varied between the interventions. No studies of alprazolam, lorazepam, or etizolam met the inclusion criteria. The study of flunitrazepam could not be connected to the efficacy network and was only connected to 1 safety network. The single brotizolam study was old and sparsely reported, ${ }^{53}$ therefore the efficacy results for brotizolam should be considered cautiously.

Longer-term data were available for few interventions (lemborexant, suvorexant, zolpidem-IR, eszopiclone, and ramelteon).

\section{Conclusions}

Lemborexant was ranked highest of the treatments studied on 3 out of the 4 objectively measured insomnia efficacy outcomes, with a safety profile broadly similar to other treatments with regard to SAEs and withdrawals due to AEs. The highest-ranked intervention exceeded clinical significance thresholds. Treatment effect was similar in older and adult populations.

\section{DISCLOSURES}

This work was funded by Eisai Inc., which was involved with all stages of the study and analysis. McElroy, O'Leary, and Adena are consultants with Datalytics Pty Ltd., which was paid by Eisai Inc. for conducting the literature review and analysis. They were not financially compensated for collaborative efforts on publication-related activities. Campbell, Tahami Monfared, and Meier are employed by Eisai Inc.

This study was presented as a poster at AMCP Nexus Virtual, October 20-23, 2020 and at the AGS Virtual Annual Scientific Meeting 2021, May 13-15, 2021.

\section{ACKNOWLEDGMENTS}

The authors thank Margaret Moline, PhD (Eisai Inc.), for interpretation of clinical results. The authors received editorial support from Carole Nadin (Fleetwith Ltd., funded by Eisai Inc.).

\section{REFERENCES}

1. Roth T, Coulouvrat C, Hajak G, et al. Prevalence and perceived health associated with insomnia based on DSM-IV-TR; International Statistical Classification of Diseases and Related Health Problems, Tenth Revision; and Research Diagnostic Criteria/International Classification of Sleep Disorders, Second Edition criteria: results from the America Insomnia Survey. Biol Psychiatry. 2011;69(6):592-600.

2. Olfson M, Wall M, Liu SM, Morin CM, Blanco C. Insomnia and impaired quality of life in the United States. J Clin Psychiatry. 2018;79(5):17m12020.

3. Patel D, Steinberg J, Patel P. Insomnia in the elderly: a review. J Clin Sleep Med. 2018;14(6):1017-24.

4. Connor J, Norton R, Ameratunga S, et al. Driver sleepiness and risk of serious injury to car occupants: population based case control study. BMJ. 2002;324(7346):1125.

5. Lombardi DA, Folkard S, Willetts JL, Smith GS. Daily sleep, weekly working hours, and risk of work-related injury: US National Health Interview Survey (20042008). Chronobiol Int. 2010;27(5):1013-30.

6. Leger D, Bayon V, Ohayon MM, et al. Insomnia and accidents: cross-sectional study (EQUINOX) on sleep-related home, work and car accidents in 5293 subjects with insomnia from 10 countries. J Sleep Res. 2014;23(2):143-52.

7. Stone KL, Blackwell TL, Ancoli-Israel S, et al. Sleep disturbances and risk of falls in older community-dwelling men: the outcomes of Sleep Disorders in Older Men (MrOS Sleep) Study. J Am Geriatr Soc. 2014;62(2):299-305.

8. Leger D, Morin CM, Uchiyama M, Hakimi Z, Cure S, Walsh JK. Chronic insomnia, quality-of-life, and utility scores: comparison with good sleepers in a cross-sectional international survey. Sleep Med. 2012;13(1):43-51.

9. Riemann D, Baglioni C, Bassetti C, et al. European guideline for the diagnosis and treatment of insomnia. J Sleep Res. 2017;26(6):675-700. 
10. Belsomra. Prescribing information. Merck and Co Inc; 2020. Accessed April 6, 2020. https://www.accessdata.fda.gov/drugsatfda_docs/ label/2020/204569s006lbl.pdf

11. Herring WJ, Connor KM, Ivgy-May N, et al. Suvorexant in patients with insomnia: results from two 3-month randomized controlled clinical trials. Biol Psychiatry. 2016;79(2):136-48.

12. Brewster GS, Riegel B, Gehrman PR. Insomnia in the older adult. Sleep Med Clin. 2018;13(1):13-19.

13. Mendelson WB. A review of the evidence for the efficacy and safety of trazodone in insomnia. J Clin Psychiatry. 2005;66(4):469-76.

14. Dayvigo. Prescribing information. Eisai Inc; 2019. Accessed February 10, 2020. https://www.accessdata.fda.gov/drugsatfda docs/ label/2019/212028s000lbl.pdf

15. Karppa M, Yardley J, Pinner K, et al. Long-term efficacy and tolerability of lemborexant compared with placebo in adults with insomnia disorder: results from the phase 3 randomized clinical trial SUNRISE 2. Sleep. 2020;43(9):zsaa123.

16. Rosenberg R, Murphy P, Zammit G, et al. Comparison of lemborexant with placebo and zolpidem tartrate extended release for the treatment of older adults with insomnia disorder: a phase 3 randomized clinical trial. JAMA Netw Open. 2019;2(12):e1918254.

17. Buscemi N, Vandermeer B, Friesen C, et al. The efficacy and safety of drug treatments for chronic insomnia in adults: a meta-analysis of RCTs. J Gen Intern Med. 2007;22(9):1335-50.

18. Huedo-Medina TB, Kirsch I, Middlemass J, Klonizakis M, Siriwardena AN. Effectiveness of non-benzodiazepine hypnotics in treatment of adult insomnia: meta-analysis of data submitted to the Food and Drug Administration. BMJ. 2012;345:e8343.

19. Winkler A, Auer C, Doering BK, Rief W. Drug treatment of primary insomnia: a meta-analysis of polysomnographic randomized controlled trials. CNS Drugs. 2014;28(9):799-816.
20. Agency for Healthcare Research and Quality (AHRQ). Management of insomnia disorder in adults: current state of the evidence. Clinician summary. August 1, 2017. Accessed May 22, 2019. https:// effectivehealthcare.ahrq.gov/products/ insomnia/clinician

21. Sateia MJ, Buysse DJ, Krystal AD, Neubauer DN, Heald JL. Clinical practice guideline for the pharmacologic treatment of chronic insomnia in adults: an American Academy of Sleep Medicine Clinical Practice Guideline. J Clin Sleep Med. 2017;13(2):307-49.

22. Rosner S, Englbrecht C, Wehrle R, Hajak G, Soyka M. Eszopiclone for insomnia. Cochrane Database Syst Rev. 2018;10:(10):CD010703.

23. Kishi T, Nomura I, Matsuda Y, et al. Lemborexant vs suvorexant for insomnia: a systematic review and network metaanalysis. J Psychiatr Res. 2020;128:68-74.

24. Bastien CH, Vallieres A, Morin CM. Validation of the Insomnia Severity Index as an outcome measure for insomnia research. Sleep Med. 2001;2(4):297-307.

25. Thorlund K, Walter SD, Johnston BC, Furukawa TA, Guyatt GH. Pooling health-related quality of life outcomes in meta-analysis-a tutorial and review of methods for enhancing interpretability. Res Synth Methods. 2011;2(3):188-203.

26. Wan X, Wang W, Liu J, Tong T.

Estimating the sample mean and standard deviation from the sample size, median, range and/or interquartile range. BMC Med Res Methodol. 2014;14:135.

27. Follmann D, Elliott P, Suh I, Cutler J. Variance imputation for overviews of clinical trials with continuous response. J Clin Epidemiol. 1992;45(7):769-73.

28. National Institute for Health and Care Excellence (NICE). Single technology appraisal: user guide for company evidence submission template [PMG24]. 2017. Accessed April 16, 2019. https://www.nice. org.uk/process/pmg24/chapter/clinicaleffectiveness \#quality-assessment-of-the$\underline{\text { relevant-clinical-effectiveness-evidence }}$
29. Dias S, Sutton AJ, Ades AE, Welton NJ. Evidence synthesis for decision making 2: a generalized linear modeling framework for pairwise and network meta-analysis of randomized controlled trials. Med Decis Making. 2013;33(5):607-17.

30. Lunn DJ, Thomas A, Best N, Spiegelhalter D. WinBUGS - a Bayesian modelling framework: concepts, structure, and extensibility. Statistics and Computing. 2000;10:325-37. Accessed April 29, 2021. https://www.mrc-bsu.cam. ac.uk/software/bugs/

31. StataCorp. 2017. Stata Statistical Software: Release 15. College Station,TX: StataCorp LLC.

32. White IR. Network meta-analysis. Stata J. 2015;15(4):951-85.

33. Chaimani A, Higgins JP, Mavridis D, Spyridonos P, Salanti G. Graphical tools for network meta-analysis in STATA. PLoS One. 2013;8(10):e76654.

34. Egger M, Davey Smith G, Schneider M, Minder C. Bias in meta-analysis detected by a simple, graphical test. BMJ.

1997;315(7109):629-34.

35. Dias S, Sutton AJ, Welton NJ, Ades AE. Evidence synthesis for decision making 3: heterogeneity-subgroups, meta-regression, bias, and bias-adjustment. Med Decis Making. 2013;33(5):618-40.

36. Ancoli-Israel S, Walsh JK, Mangano RM, Fujimori M. Zaleplon. A novel nonbenzodiazepine hypnotic, effectively treats insomnia in elderly patients without causing rebound effects. Prim Care Companion J Clin Psychiatry. 1999;1(4):114-20.

37. Ancoli-Israel S, Krystal AD, McCall $\mathrm{WV}$, et al. A 12-week, randomized, doubleblind, placebo-controlled study evaluating the effect of eszopiclone $2 \mathrm{mg}$ on sleep/ wake function in older adults with primary and comorbid insomnia. Sleep. 2010;33(2):225-34.

38. Black J, Pillar G, Hedner J, et al. Efficacy and safety of almorexant in adult chronic insomnia: a randomized placebocontrolled trial with an active reference. Sleep Med. 2017;36:86-94. 
39. Dehlin O, Rubin B, Rundgren A.

Double-blind comparison of zopiclone and flunitrazepam in elderly insomniacs with special focus on residual effects. Curr Med Res Opin. 1995;13(6):317-24.

40. Dorsey CM, Lee KA, Scharf MB. Effect of zolpidem on sleep in women with perimenopausal and postmenopausal insomnia: a 4-week, randomized, multicenter, double-blind, placebo-controlled study. Clin Ther. 2004;26(10):1578-86.

41. Elie R, Ruther E, Farr I, Emilien G, Salinas E. Sleep latency is shortened during 4 weeks of treatment with zaleplon, a novel nonbenzodiazepine hypnotic. Zaleplon Clinical Study Group. J Clin Psychiatry. 1999;60(8):536-44.

42. Fry J, Scharf M, Mangano R, Fujimori M. Zaleplon improves sleep without producing rebound effects in outpatients with insomnia. Zaleplon Clinical Study Group. Int Clin Psychopharmacol. 2000;15(3):141-52.

43. Hajak G, Clarenbach P, Fischer W, Haase W, Ruther E. Zopiclone improves sleep quality and daytime well-being in insomniac patients: comparison with triazolam, flunitrazepam and placebo. Int Clin Psychopharmacol. 1994;9(4):251-61.

44. Hajak G, Hedner J, Eglin M, et al. A 2-week efficacy and safety study of gaboxadol and zolpidem using electronic diaries in primary insomnia outpatients. Sleep Med. 2009;10(7):705-12.

45. Hedner J, Yaeche R, Emilien G, Farr I, Salinas E. Zaleplon shortens subjective sleep latency and improves subjective sleep quality in elderly patients with insomnia. The Zaleplon Clinical Investigator Study Group. Int J Geriatr Psychiatry. 2000;15(8):704-12.

46. Krystal AD, Walsh JK, Laska E, et al. Sustained efficacy of eszopiclone over 6 months of nightly treatment: results of a randomized, double-blind, placebocontrolled study in adults with chronic insomnia. Sleep. 2003;26(7):793-9.

47. Lahmeyer H, Wilcox CS, Kann J, Leppik I. Subjective efficacy of zolpidem in outpatients with chronic insomnia: a double-blind comparison with placebo. Clin Drug Investig. 1997;13(3):134-44.
48. Leppik IE, Roth-Schechter GB, Gray GW, Cohn MA, Owens D. Doubleblind, placebo-controlled comparison of zolpidem, triazolam, and temazepam in elderly patients with insomnia. Drug Dev Res. 1997;40(3):230-38.

49. Mayer G, Wang-Weigand S, Roth-Schechter B, Lehmann R, Staner

C, Partinen M. Efficacy and safety of 6-month nightly ramelteon administration in adults with chronic primary insomnia. Sleep. 2009;32(3):351-60.

50. McCall WV, Erman M, Krystal AD, et al. A polysomnography study of eszopiclone in elderly patients with insomnia. Curr Med Res Opin. 2006;22(9):1633-42.

51. Pinto LR Jr, Bittencourt LR, Treptow EC, Braga LR, Tufik S. Eszopiclone versus zopiclone in the treatment of insomnia. Clinics (Sao Paulo). 2016;71(1):5-9.

52. Randall S, Roehrs TA, Roth T. Efficacy of eight months of nightly zolpidem: a prospective placebo-controlled study. Sleep. 2012;35(11):1551-7.

53. Rickels K, Morris RJ, Mauriello R, et al. Brotizolam, a triazolothienodiazepine, in insomnia. Clin Pharmacol Ther. 1986;40(3):293-9.

54. Rosenberg J, Ahlstrom F. Randomized, double blind trial of zolpidem $10 \mathrm{mg}$ versus triazolam $0.25 \mathrm{mg}$ for treatment of insomnia in general practice. Scand J Prim Health Care. 1994;12(2):88-92.

55. Roth T, Seiden D, Sainati S, Wang-Weigand S, Zhang J, Zee P. Effects of ramelteon on patient-reported sleep latency in older adults with chronic insomnia. Sleep Med. 2006;7(4):312-8.

56. Roth T, Soubrane C, Titeux L, Walsh JK, Zoladult Study G. Efficacy and safety of zolpidem-MR: a doubleblind, placebo-controlled study in adults with primary insomnia. Sleep Med. 2006;7(5):397-406.

57. Scharf MB, Roth PB, Dominguez RA, Ware JC. Estazolam and flurazepam: a multicenter, placebo-controlled comparative study in outpatients with insomnia. J Clin Pharmacol. 1990;30(5):461-7.
58. Scharf MB, Roth T, Vogel GW, Walsh JK. A multicenter, placebo-controlled study evaluating zolpidem in the treatment of chronic insomnia. J Clin Psychiatry. 1994;55(5):192-9.

59. Scharf M, Erman M, Rosenberg R, et al. A 2-week efficacy and safety study of eszopiclone in elderly patients with primary insomnia. Sleep. 2005;28(6):720-7.

60. Murphy P, Moline M, Mayleben D, et al. Lemborexant, a dual orexin receptor antagonist (DORA) for the treatment of insomnia disorder: results from a Bayesian, adaptive, randomized, doubleblind, placebo-controlled study. J Clin Sleep Med. 2017;13(11):1289-99.

61. Yardley J, Pinner K, Murphy P, Filippov G, Zammit G, Moline M. Efficacy of lemborexant compared with placebo in adult and elderly subjects with insomnia. Poster presented at Advances in Sleep and Circadian Science (ASCS)/Sleep Research Society (SRS); February 1-4, 2019; Clearwater, FL.

62. Soares CN, Joffe H, Rubens R, Caron J, Roth T, Cohen L. Eszopiclone in patients with insomnia during perimenopause and early postmenopause: a randomized controlled trial. Obstet Gynecol. 2006;108(6):1402-10.

63. Tsutsui S, Zolipidem Study Group. A double-blind comparative study of zolpidem versus zopiclone in the treatment of chronic primary insomnia. J Int Med Res. 2001;29(3):163-77.

64. Uchimura N, Ogawa A, Hamamura M, Hashimoto T, Nagata H, Uchiyama M. Efficacy and safety of ramelteon in Japanese adults with chronic insomnia: a randomized, double-blind, placebocontrolled study. Expert Rev Neurother. 2011;11(2):215-24.

65. Uchiyama M, Hamamura M, Kuwano T, Nishiyama H, Nagata H, Uchimura N. Evaluation of subjective efficacy and safety of ramelteon in Japanese subjects with chronic insomnia. Sleep Med. 2011;12(2):119-26. 
66. Voshaar RC, van Balkom AJ, Zitman FG. Zolpidem is not superior to temazepam with respect to rebound insomnia: a controlled study. Eur Neuropsychopharmacol. 2004;14(4):301-06.

67. Walsh J, Targum SD, Pegram V, et al. A multi-center clinical investigation of estazolam: short-term efficacy. Curr Ther Res Clin Exp. 1984;36(5):866-74.

68. Walsh JK, Erman M, Erwin CW, et al. Subjective hypnotic efficacy of trazodone and zolpidem in DSMIII-R primary insomnia. Human Psychopharmacology. 1998;13(3):191-98.

69. Walsh JK, Fry J, Erwin CW, Scharf M, Roth T, Vogel GW. Efficacy and tolerability of 14-day administration of zaleplon $5 \mathrm{mg}$ and $10 \mathrm{mg}$ for the treatment of primary insomnia. Clin Drug Investig. 1998;16(5):347-54.

70. Walsh JK, Vogel GW, Scharf M, et al. A five week, polysomnographic assessment of zaleplon $10 \mathrm{mg}$ for the treatment of primary insomnia. Sleep Med. 2000;1(1):41-49.
71. Walsh JK, Krystal AD, Amato DA, et al. Nightly treatment of primary insomnia with eszopiclone for six months: effect on sleep, quality of life, and work limitations. Sleep. 2007;30(8):959-68.

72. Walsh JK, Soubrane C, Roth T. Efficacy and safety of zolpidem extended release in elderly primary insomnia patients. Am J Geriatr Psychiatry. 2008;16(1):44-57.

73. Wang-Weigand S, Watissee M, Roth T. Use of a post-sleep questionnaire-interactive voice response system (PSQ-IVRS) to evaluate the subjective sleep effects of ramelteon in adults with chronic insomnia. Sleep Med. 2011;12(9):920-3.

74. Ware JC, Walsh JK, Scharf MB, Roehrs T, Roth T, Vogel GW. Minimal rebound insomnia after treatment with 10-mg zolpidem. Clin Neuropharmacol. 1997;20(2):116-25.

75. Yan X, Huang S, Ma C, et al. A randomized, double-blind, double-dummy, multicenter, controlled trial on brotizolam intervention in outpatients with insomnia. Int J Psychiatry Clin Pract. 2013;17(4):239-43.
76. Zammit GK, McNabb LJ, Caron J, Amato DA, Roth T. Efficacy and safety of eszopiclone across 6-weeks of treatment for primary insomnia. Curr Med Res Opin. 2004;20(12):1979-91.

77. Zammit G, Erman M, Wang-Weigand S, Sainati S, Zhang J, Roth T. Evaluation of the efficacy and safety of ramelteon in subjects with chronic insomnia. J Clin Sleep Med. 2007;3(5):495-504.

78. Moline M, Zammit G, Yardley J, et al. Lack of residual morning effects of lemborexant treatment for insomnia: summary of findings across 9 clinical trials. Postgrad Med. 2020:1-11.

79. Lunesta. Prescribing information. Sunovion Pharmaceuticals Inc. 2014. Accessed April 6, 2020. https://www. accessdata.fda.gov/drugsatfda docs/ label/2014/021476s030lbl.pdf

80. Rosenberg R, Murphy P, Chou C, Dhadda S, Zammit G, Moline M.

Comparison of lemborexant with zolpidem extended release and placebo: topline results from a phase 3 study in subjects 55 years and older with insomnia. J Sleep Res. 2018;27:165. 